PROCEEDINGS OF THE

AMERICAN MATHEMATICAL SOCIETY

Volume 134, Number 3, Pages 881-891

S 0002-9939(05)08034-2

Article electronically published on July 7, 2005

\title{
o-BOUNDED GROUPS AND OTHER TOPOLOGICAL GROUPS WITH STRONG COMBINATORIAL PROPERTIES
}

\author{
BOAZ TSABAN \\ (Communicated by Alan Dow)
}

\begin{abstract}
We construct several topological groups with very strong combinatorial properties. In particular, we give simple examples of subgroups of $\mathbb{R}$ (thus strictly o-bounded) which have the Menger and Hurewicz properties but are not $\sigma$-compact, and show that the product of two $o$-bounded subgroups of $\mathbb{R}^{\mathbb{N}}$ may fail to be $o$-bounded, even when they satisfy the stronger property $\mathrm{S}_{1}\left(\mathcal{B}_{\Omega}, \mathcal{B}_{\Omega}\right)$. This solves a problem of Tkačenko and Hernandez, and extends independent solutions of Krawczyk and Michalewski and of Banakh, Nickolas, and Sanchis. We also construct separable metrizable groups $G$ of size continuum such that every countable Borel $\omega$-cover of $G$ contains a $\gamma$-cover of $G$.
\end{abstract}

\section{INTRODUCTION}

In [15, 11, a unified framework for topological diagonalizations is established, which turns out to be closely related to several notions which appear in a recently flourishing study of topological groups in terms of their covering properties (see, e.g., [18, 9, 10, 12, 13, and references therein). A comprehensive study of these interrelations is currently being carried out by Babinkostova, Kočinac, and Scheepers 1]. The purpose of this paper is to adopt several recent construction techniques from the general theory of topological diagonalizations to the theory of topological groups.

1.1. Topological diagonalizations. We briefly describe the general framework. Let $X$ be a topological space. An open cover $\mathcal{U}$ of $X$ is an $\omega$-cover of $X$ if $X$ is not in $\mathcal{U}$, and for each finite subset $F$ of $X$, there is a set $U \in \mathcal{U}$ such that $F \subseteq U$. $\mathcal{U}$ is a $\gamma$-cover of $X$ if it is infinite and for each $x$ in $X, x \in U$ for all but finitely many $U \in \mathcal{U}$. Let $\mathcal{O}, \Omega$, and $\Gamma$ denote the collections of all countable open covers, $\omega$-covers, and $\gamma$-covers of $X$, respectively. Let $\mathcal{A}$ and $\mathcal{B}$ be collections of covers of a space $X$. Following are selection hypotheses which $X$ might satisfy or not satisfy:

$\mathrm{S}_{1}(\mathcal{A}, \mathcal{B})$ : For each sequence $\left\{\mathcal{U}_{n}\right\}_{n \in \mathbb{N}}$ of members of $\mathcal{A}$, there exist members $U_{n} \in \mathcal{U}_{n}, n \in \mathbb{N}$, such that $\left\{U_{n}\right\}_{n \in \mathbb{N}} \in \mathcal{B}$.

$\mathrm{S}_{\text {fin }}(\mathcal{A}, \mathcal{B})$ : For each sequence $\left\{\mathcal{U}_{n}\right\}_{n \in \mathbb{N}}$ of members of $\mathcal{A}$, there exist finite (possibly empty) subsets $\mathcal{F}_{n} \subseteq \mathcal{U}_{n}, n \in \mathbb{N}$, such that $\bigcup_{n \in \mathbb{N}} \mathcal{F}_{n} \in \mathcal{B}$.

Received by the editors July 8, 2003 and, in revised form, September 20, 2004.

2000 Mathematics Subject Classification. Primary 54H11; Secondary 37F20.

Key words and phrases. o-bounded groups, $\gamma$-sets, Luzin sets, selection principles.

(C)2005 American Mathematical Society 
$\bigcup_{\text {fin }}(\mathcal{A}, \mathcal{B})$ : For each sequence $\left\{\mathcal{U}_{n}\right\}_{n \in \mathbb{N}}$ of members of $\mathcal{A}$ which do not contain a finite subcover, there exist finite (possibly empty) subsets $\mathcal{F}_{n} \subseteq \mathcal{U}_{n}$, $n \in \mathbb{N}$, such that $\left\{\bigcup \mathcal{F}_{n}\right\}_{n \in \mathbb{N}} \in \mathcal{B}$.

Some of the properties defined in this manner were studied earlier by Hurewicz $\left(\mathrm{U}_{\text {fin }}(\mathcal{O}, \Gamma)\right)$, Menger $\left(\mathrm{S}_{\text {fin }}(\mathcal{O}, \mathcal{O})\right)$, Rothberger $\left(\mathrm{S}_{1}(\mathcal{O}, \mathcal{O})\right.$, traditionally known as the $C^{\prime \prime}$ property), Gerlits and Nagy $\left(\mathrm{S}_{1}(\Omega, \Gamma)\right.$, traditionally known as the $\gamma$-property), and others. Many equivalences hold among these properties, and the surviving ones appear in Figure 1 (where an arrow denotes implication), to which no arrow can be added except perhaps from $\mathrm{U}_{\text {fin }}(\mathcal{O}, \Gamma)$ or $\mathrm{U}_{\text {fin }}(\mathcal{O}, \Omega)$ to $\mathrm{S}_{\text {fin }}(\Gamma, \Omega)$ [11.

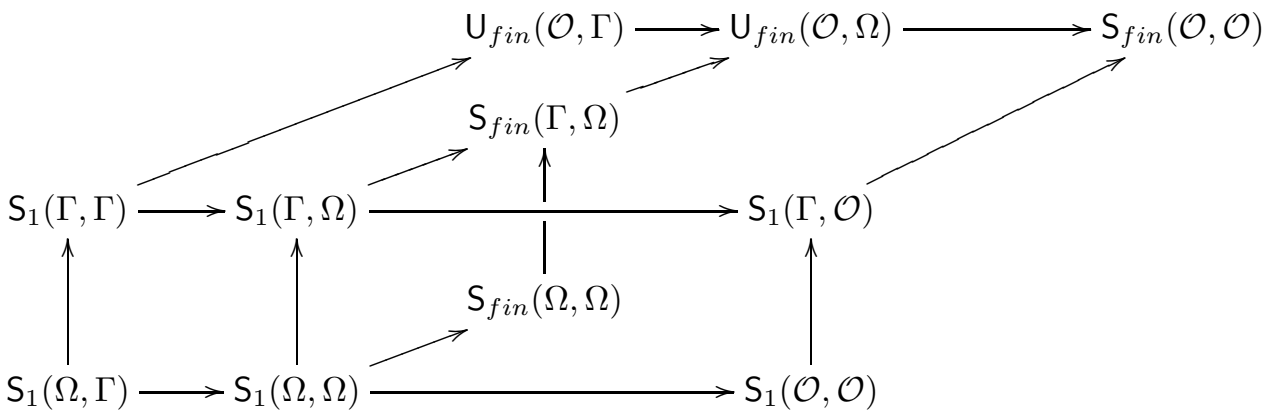

Figure 1. The Scheepers Diagram

Each selection principle has a naturally associated game, but we will restrict our attention to the game $\mathrm{G}_{\text {fin }}(\mathcal{A}, \mathcal{B})$, which is played as follows: In the $n$th inning, ONE chooses an element $\mathcal{U}_{n}$ of $\mathcal{A}$ and then TWO responds by choosing a finite subset $\mathcal{F}_{n}$ of $\mathcal{U}_{n}$. They play an inning per natural number. A play $\left(\mathcal{U}_{0}, \mathcal{F}_{0}, \mathcal{U}_{1}, \mathcal{F}_{1}, \ldots\right)$ is won by TWO if $\bigcup_{n} \mathcal{F}_{n} \in \mathcal{B}$; otherwise ONE wins. We will write $\mathrm{ONE} \uparrow \mathrm{G}_{\text {fin }}(\mathcal{A}, \mathcal{B})$ (respectively, TWO $\uparrow \mathrm{G}_{f i n}(\mathcal{A}, \mathcal{B})$ ) for "ONE (respectively, TWO) has a winning strategy in $\mathrm{G}_{\text {fin }}(\mathcal{A}, \mathcal{B})$ ".

1.2. o-bounded groups. Okunev introduced the following notion as an approximation of $\sigma$-compact groups: A topological group $G$ is o-bounded if for each sequence $\left\{U_{n}\right\}_{n \in \mathbb{N}}$ of neighborhoods of the unit element of $G$, there exists a sequence $\left\{F_{n}\right\}_{n \in \mathbb{N}}$ of finite subsets of $G$ such that $G=\bigcup_{n} F_{n} \cdot U_{n}$. It is possible to state this definition in the language of selection principles. Let $\mathcal{O}_{\text {nbd }}$ denote the covers of $G$ of the form $\{g \cdot U: g \in G\}$, where $U$ is a neighborhood of the unit element of $G$. Then $G$ is $o$-bounded if, and only if, $G$ satisfies $\mathrm{S}_{\text {fin }}\left(\mathcal{O}_{\text {nbd }}, \mathcal{O}\right)$.

According to Tkačenko, a topological group $G$ is strictly o-bounded if TWO has a winning strategy in the game $\mathrm{G}_{\text {fin }}\left(\mathcal{O}_{\mathrm{nbd}}, \mathcal{O}\right)$. Clearly, every subgroup of a $\sigma$-compact group is strictly $o$-bounded, but the converse does not hold [9].

Notational convention. For sets $X, Y,{ }^{X} Y$ denotes the collection of all functions from $X$ to $Y$. If $Y$ is a topological space, then the topology on ${ }^{X} Y$ is the Tychonoff product topology. 


\section{Two Almost $\sigma$-COMPACT SUBgRoups OF $\mathbb{R}$}

The Baire space ${ }^{\mathbb{N}} \mathbb{N}$ is (quasi)ordered by eventual dominance: $f \leq^{*} g$ if $f(n) \leq$ $g(n)$ for all but finitely many $n$. A subset of ${ }^{\mathbb{N}}$ is dominating if it is cofinal in ${ }^{\mathbb{N}}$ with respect to $\leq^{*}$. If a subset of ${ }^{\mathbb{N}}$ is unbounded with respect to $\leq^{*}$, then we simply say that it is unbounded. Let $\mathfrak{b}$ (respectively, $\mathfrak{d}$ ) denote the minimal cardinality of an unbounded (respectively, dominating) subset of ${ }^{\mathbb{N}}$.

We use the following setting from [5]. Let $\overline{\mathbb{N}}=\mathbb{N} \cup\{\infty\}$ be the one point compactification of $\mathbb{N}$. (A subset $A \subseteq \overline{\mathbb{N}}$ is open if $A \subseteq \mathbb{N}$, or $\infty \in A$ and $A$ is cofinite.) Let ${ }^{\mathbb{N}} / \overline{\mathbb{N}} \subseteq \mathbb{N} \overline{\mathbb{N}}$ consist of the nondecreasing functions $f \in{ }^{\mathbb{N}} \overline{\mathbb{N}}$. ${ }^{\mathbb{N}} / \overline{\mathbb{N}}$ is homeomorphic to the Cantor set of reals. For each increasing finite sequence $s$ of natural numbers, let $q_{s} \in \mathbb{N} / \overline{\mathbb{N}}$ be defined by $q_{s}(k)=s(k)$ if $k<|s|$, and $q_{s}(k)=\infty$ otherwise. Let $Q$ be the collection of all these elements $q_{s}$.

Theorem 1. There exists a non- $\sigma$-compact subgroup $G_{H}$ of $\mathbb{R}$ of cardinality $\mathfrak{b}$ such that all finite powers of $G_{H}$ satisfy $\mathrm{U}_{\text {fin }}(\mathcal{O}, \Gamma)$ (in particular, they satisfy $\left.\mathrm{S}_{\text {fin }}(\Omega, \Omega)\right)$.

Proof. Let $B=\left\{f_{\alpha}: \alpha<\mathfrak{b}\right\} \subseteq \mathbb{N}^{N}$ be a $\leq^{*}$-unbounded set of strictly increasing elements of ${ }^{\mathbb{N}} \mathbb{N}$ which forms a $\mathfrak{b}$-scale (that is, for each $\alpha<\beta, f_{\alpha} \leq^{*} f_{\beta}$ ), and set $H=B \cup Q$. In [5] it is proved that all finite powers of $H$ satisfy $\mathrm{U}_{\text {fin }}(\mathcal{O}, \Gamma)$.

Think of $H$ as a set of real numbers. For each $n$, the set

$$
G_{n}=\left\{\alpha_{1} g_{1}+\cdots+\alpha_{n} g_{n}: \alpha_{1}, \ldots, \alpha_{n} \in \mathbb{Z}, g_{1}, \ldots, g_{n} \in H\right\}
$$

is a union of countably many continuous images of $H^{n}$, thus for each $k,\left(G_{n}\right)^{k}$ is a union of countably many continuous images of $H^{n k}$. As the property $\mathrm{U}_{\text {fin }}(\mathcal{O}, \Gamma)$ is preserved under taking continuous images and countable unions [11, 4, we have that each set $\left(G_{n}\right)^{k}$ satisfies $\mathrm{U}_{\text {fin }}(\mathcal{O}, \Gamma)$.

Take $G_{H}=\langle H\rangle$. Then $G_{H}=\bigcup_{n \in \mathbb{N}} G_{n}$ and for each $n, G_{n} \subseteq G_{n+1}$. Thus, $\left(G_{H}\right)^{k}=\bigcup_{n \in \mathbb{N}}\left(G_{n}\right)^{k}$ for each $k$; therefore $\left(G_{H}\right)^{k}$ satisfies $\bigcup_{f i n}(\mathcal{O}, \Gamma)$ for each $k$. Now, satisfying $\mathrm{U}_{\text {fin }}(\mathcal{O}, \mathcal{O})$ in all finite powers implies $\mathrm{S}_{f i n}(\Omega, \Omega)[1]$.

It was observed by Pol and Zdomsky that one can make sure that $G_{H}$ is not $\sigma$ compact by embedding ${ }^{\mathbb{N}} / \overline{\mathbb{N}}$ in a Cantor set of reals, $C$, that is linearly independent over $\mathbb{Q}$. This way, $H=\langle H\rangle \cap C$ is a closed subset of $\langle H\rangle=G_{H}$. Since $H$ is not $\sigma$-compact [5], we get that $G_{H}$ cannot be $\sigma$-compact.

Problem 2. Does $G_{H}$ satisfy $\mathrm{S}_{1}(\Gamma, \Gamma)$ ?

Corollary 3. TWO $\uparrow \mathrm{G}_{\text {fin }}(\mathcal{O}, \mathcal{O})$ is strictly stronger than $T W O \uparrow \mathrm{G}_{\text {fin }}\left(\mathcal{O}_{\mathrm{nbd}}, \mathcal{O}\right)$ (strict o-boundedness).

Proof. By a well-known theorem of Telgársky, TWO $\uparrow \mathrm{G}_{f i n}(\mathcal{O}, \mathcal{O})$ if, and only if, the space $G$ is $\sigma$-compact. Contrast this with Theorem 1 .

Theorem 1 has a group-theoretic consequence.

Corollary 4. Assume that for each $m,\left\{g_{m, n}+U_{m}\right\}_{n \in \mathbb{N}}$ is a cover of $G_{H}$. Then there exists a sequence $\left\{m_{n}\right\}_{n \in \mathbb{N}}$ such that

$$
G_{H}=\bigcup_{k} \bigcap_{n>k}\left(\left\{g_{n, 1}, \ldots, g_{n, m_{n}}\right\}+U_{n}\right) \text {. }
$$

In fact, this property is satisfied by all finite powers of $G_{H}$. 
Let $D=\left\{g_{\alpha}: \alpha<\mathfrak{d}\right\}$ be a dominating subset of ${ }^{\mathbb{N}} \mathbb{N}$ where each $g_{\alpha}$ is increasing, and for each $f \in{ }^{\mathbb{N}} \mathbb{N}$ there exists $\alpha_{0}<\mathfrak{d}$ such that for any finite set $F \subseteq \mathfrak{d} \backslash \alpha_{0}$, $f(n)<\min \left\{g_{\beta}(n): \beta \in F\right\}$ for infinitely many $n$. Such a set was constructed in [5.

A subset $F$ of ${ }^{\mathbb{N}} \mathbb{N}$ is finitely-dominating if for each $g \in{ }^{\mathbb{N}} \mathbb{N}$ there exist $k$ and $f_{1}, \ldots, f_{k} \in{ }^{\mathbb{N}} \mathbb{N}$ such that $g(n) \leq * \max \left\{f_{1}(n), \ldots, f_{k}(n)\right\}$. For conciseness, we use the following shortened notation:

Ax: Either a union of less than $\mathfrak{d}$ many not dominating sets is not dominating (in other words, $\mathfrak{b}=\mathfrak{d}$ ), or else a union of less than $\mathfrak{d}$ many not finitelydominating sets of increasing functions is not finitely-dominating.

In [5] it is shown that $\mathrm{Ax}$ implies that $M=D \cup Q$ satisfies $\mathrm{S}_{\text {fin }}(\Omega, \Omega$ ). (Observe that $\mathrm{S}_{\text {fin }}(\Omega, \Omega)$ is preserved under taking finite powers [11.) Assuming $\mathrm{Ax}$, one shows as in Theorem 1 that all finite powers of $G_{M}=\langle M\rangle$ satisfy $\mathrm{U}_{f i n}(\mathcal{O}, \mathcal{O})$ and obtains the following.

Theorem 5. Assume that Ax holds. Then:

(1) There exists a non- $\sigma$-compact subgroup $G_{M}$ of $\mathbb{R}$ of cardinality $\mathfrak{d}$ such that $G_{M}$ satisfies $\mathrm{S}_{\text {fin }}(\Omega, \Omega)$.

(2) Assume that for each $m,\left\{g_{m, n}+U_{m}\right\}_{n \in \mathbb{N}}$ is a cover of $G_{M}$. Then there exists a sequence $\left\{m_{n}\right\}_{n \in \mathbb{N}}$ such that for each finite subset $F$ of $G_{M}$, there exists $n$ such that $F \subseteq\left\{g_{n, 1}, \ldots, g_{n, m_{n}}\right\}+U_{n}$. Moreover, this holds for all finite powers of $G_{M}$.

It follows from the next section that the hypothesis $A x$ is not necessary to prove Theorem 5 (namely, it also follows from the incomparable assumption $\operatorname{cov}(\mathcal{M})=\mathfrak{c}$ ).

Problem 6. Is Theorem 5 provable in ZFC?

\section{Products of o-Bounded Groups}

In Problem 3.2 of [18 and Problem 5.2 of 9 ] it is asked whether the (Tychonoff) product of two o-bounded groups is o-bounded. We give a negative answer. A negative answer was independently given by Krawczyk and Michalewski [13, but our result is stronger in the following sense: Let $G$ be a topological group such that all finite powers of $G$ are Lindelöf. Then each open $\omega$-cover of $G$ contains a countable $\omega$-cover of $G$. Let $\mathcal{B}_{\Omega}$ denote the collection of all countable Borel $\omega$-covers of $G$. In this case,

$$
\mathrm{S}_{1}\left(\mathcal{B}_{\Omega}, \mathcal{B}_{\Omega}\right) \rightarrow \mathrm{S}_{1}(\Omega, \Omega) \rightarrow \mathrm{S}_{\text {fin }}(\mathcal{O}, \mathcal{O}),
$$

where no implication can be reversed [11, 17, and the last property (the Menger property) implies $S_{f i n}\left(\mathcal{O}_{\text {nbd }}, \mathcal{O}\right)$ (o-boundedness). In [13] it is proved that, assuming $\operatorname{cov}(\mathcal{M})=\mathfrak{c}$ (this is a small portion of the Continuum Hypothesis), there exist groups $G_{1}$ and $G_{2}$ satisfying the Menger property $\mathrm{S}_{f i n}(\mathcal{O}, \mathcal{O})$, such that $G_{1} \times G_{2}$ is not $o$-bounded. We use the same hypothesis to show that there exist such groups satisfying $\mathrm{S}_{1}\left(\mathcal{B}_{\Omega}, \mathcal{B}_{\Omega}\right)$.

Fact 7. Assume that $G$ is $a \leq^{*}$-dominating subgroup of ${ }^{\mathbb{N}} \mathbb{R}$. Then $G$ is not obounded.

Proof. As $\mathbb{N}$ can be partitioned into infinitely many infinite sets, the following holds. 
Lemma 8. For each o-bounded group $G$ and sequence $\left\{U_{n}\right\}_{n \in \mathbb{N}}$ of neighborhoods of the identity of $G$, there exists a sequence $\left\{F_{n}\right\}_{n \in \mathbb{N}}$ of finite subsets of $G$ with $G=\bigcap_{m} \bigcup_{n>m} F_{n} \cdot G$.

Consider the open sets $U_{n}=\left\{f \in{ }^{\mathbb{N}} \mathbb{R}:|f(n)|<1\right\} \subseteq{ }^{\mathbb{N}} \mathbb{R}$. For each sequence $\left\{F_{n}\right\}_{n \in \mathbb{N}}$ of finite subsets of ${ }^{\mathbb{N}} \mathbb{R}, \bigcup_{n} F_{n}+U_{n}$ is $\leq^{*}$-bounded in ${ }^{\mathbb{N}} \mathbb{R}$. Let $h \in{ }^{\mathbb{N}} \mathbb{R}$ be a witness for that. As $G$ is dominating, there exists $g \in G$ such that $h \leq^{*} g$. Then $g \notin \bigcap_{m} \bigcup_{n>m} F_{n} \cdot G$.

Let $\mathfrak{c}=|\mathbb{R}|$. The assertion $\operatorname{cov}(\mathcal{M})=\mathfrak{c}$ means that $\mathbb{R}$ (or any complete, separable, metric space) is not the union of less than continuum many meager (= first category) sets. We say that $L$ is a $\kappa$-Luzin group if $|L| \geq \kappa$, and for each meager set $M$ in $L$, $|L \cap M|<\kappa$.

Theorem 9. Assume that $\operatorname{cov}(\mathcal{M})=\mathfrak{c}$. Then there exist $\mathfrak{c}$-Luzin subgroups $L_{1}, L_{2}$ of ${ }^{\mathbb{N}} \mathbb{R}$ satisfying $\mathrm{S}_{1}\left(\mathcal{B}_{\Omega}, \mathcal{B}_{\Omega}\right)$, such that the group $L_{1} \times L_{2}$ is not o-bounded. (These Luzin groups are, in fact, linear vector spaces over $\mathbb{Q}$.)

Proof. We extend the technique of [17, 4]. We stress that there exists a much easier proof if we only require that $L_{1}$ and $L_{2}$ satisfy $\mathrm{S}_{1}(\Omega, \Omega)$; however we do not supply this easier proof to avoid repetitions.

A cover $\mathcal{U}$ of $X$ is $\omega$-fat if for each finite $F \subseteq X$ and each finite family $\mathcal{F}$ of nonempty open sets, there exists $U \in \mathcal{U}$ such that $F \subseteq U$ and for each $O \in \mathcal{F}$, $U \cap O$ is not meager. Let $\mathcal{M}$ denote the meager subsets of ${ }^{\mathbb{N}} \mathbb{R}$.

Lemma 10 (4]). Assume that $\mathcal{U}$ is an $\omega$-fat cover of a set $X \subseteq{ }^{\mathbb{N}} \mathbb{R}$. Then:

(1) $\bigcup \mathcal{U}$ is comeager.

(2) For each finite $F \subseteq G$ and finite family $\mathcal{F}$ of nonempty open sets,

$$
\mathcal{U}_{F, \mathcal{F}}:=\{U \in \mathcal{U}: F \subseteq U \text { and for each } O \in \mathcal{F}, U \cap O \notin \mathcal{M}\}
$$

is an $\omega$-fat cover of $G$. Consequently, $\bigcup \mathcal{U}_{F, \mathcal{F}}$ is comeager.

For shortness, we will say that a cover $\mathcal{U}$ of $X$ is good if: For each finite $A \subseteq$ $\mathbb{Q} \backslash\{0\}$ and finite $B \subseteq G$, the family

$$
\mathcal{U}^{A, B}:=\left\{\bigcap_{q \in A, g \in B} q(U-g): U \in \mathcal{U}\right\}
$$

is an $\omega$-fat cover of $X$. These covers allow the inductive construction hinted to in the following lemma.

Lemma 11. Assume that $\mathcal{U}$ is a good cover of a group $G \subseteq{ }^{\mathbb{N}} \mathbb{R}$. Then for each element $x$ in the intersection of all sets of the form $\bigcup\left(\mathcal{U}^{A, B}\right)_{F, \mathcal{F}}$ where the members of $\mathcal{F}$ are basic open sets, $\mathcal{U}$ is a good cover of the group $G+\mathbb{Q} x$.

Proof. Fix finite sets $A \subseteq \mathbb{Q} \backslash\{0\}$ and $B \subseteq G$. We may assume that $1 \in A$. We must show that $\mathcal{U}^{A, B}$ is an $\omega$-fat cover of $G+\mathbb{Q} x$. Let $F$ be a finite subset of $G+\mathbb{Q} x$, and let $\mathcal{F}$ be a finite family of nonempty open sets. By moving to subsets we may assume that all members of $\mathcal{F}$ are basic open sets.

Choose finite sets $\tilde{A} \subseteq \mathbb{Q} \backslash\{0\}, \tilde{B} \subseteq G$, and $\tilde{F} \subseteq G$, such that $F \subseteq(\tilde{B}+\tilde{A} x) \cup \tilde{F}$, $1 \in \tilde{A}$, and $0 \in \tilde{B}$. As $x \in \bigcup\left(\mathcal{U}^{\tilde{A}^{-1} A, B+A^{-1} \tilde{B}}\right)_{\tilde{F}, \mathcal{F}}$, there exists $U \in \mathcal{U}$ such that $x \in \tilde{V}:=\bigcap_{q \in \tilde{A}^{-1}} A, g \in B+A^{-1} \tilde{B} q(U-g), \tilde{F} \subseteq \tilde{V}$, and for each $O \in \mathcal{F}, \tilde{V} \cap O$ 
is not meager. Take $V=\bigcap_{q \in A, g \in B} q(U-g)$. Then $\tilde{V} \subseteq V$, thus $\tilde{F} \subseteq V$ and for each $O \in \mathcal{F}, V \cap O$ is not meager. Now, $x \in \tilde{V}$, thus for each $\tilde{a} \in \tilde{A}$ and $\tilde{b} \in \tilde{B}, x \in \bigcap_{q \in \tilde{a}^{-1} A, g \in B+A^{-1} \tilde{b}} q(U-g)$, thus $x \in \bigcap_{q \in A, g \in B} \tilde{a}^{-1} q\left(U-\left(g+q^{-1} \tilde{b}\right)\right)$, therefore $x \in \bigcap_{q \in A, g \in B} \tilde{a}^{-1}(q(U-g)-\tilde{b})$, thus $\tilde{a} x+\tilde{b} \in V$. This shows that $F \subseteq(\tilde{A} x+\tilde{B}) \cup \tilde{F} \subseteq V \in \mathcal{U}^{A, B}$, and we are done.

Since we are going to construct Luzin groups, the following lemma tells us that we need not consider covers which are not good.

Lemma 12. Assume that $L$ is a subgroup of $\mathbb{N} \mathbb{R}$ such that $\mathbb{Q} \cdot L \subseteq L$ and for each nonempty basic open set $O, L \cap O$ is not meager. Then every countable Borel $\omega$-cover $\mathcal{U}$ of $L$ is a good cover of $L$.

Proof. Assume that $\mathcal{U}$ is a countable collection of Borel sets which is not a good cover of $L$. Then there exist finite sets $A \subseteq \mathbb{Q} \backslash\{0\}, B \subseteq L, F \subseteq L$, and $\mathcal{F}$ of nonempty open sets such that for each $V \in \mathcal{U}^{A, B}$ containing $F, V \cap O$ is meager for some $O \in \mathcal{F}$. For each $O \in \mathcal{F}$ let

$$
M_{O}=\bigcup\left\{V \in \mathcal{U}^{A, B}: F \subseteq V \text { and } V \cap O \in \mathcal{M}\right\} .
$$

Then $M_{O} \cap O$ is meager, thus there exists $x_{O} \in(L \cap O) \backslash M_{O}$. Then $F \cup\left\{x_{O}: O \in \mathcal{F}\right\}$ is not covered by any $V \in \mathcal{U}^{A, B}$. We will show that this cannot be the case.

Put $F^{\prime}=A^{-1} F+B$. As $\mathbb{Q} \cdot L \subseteq L, F^{\prime}$ is a finite subset of $L$. Thus, there exists $U \in \mathcal{U}$ such that $F^{\prime} \subseteq U$. Consequently, for each $q \in A$ and $g \in B, x \in q(U-g)$ for each $x \in F$, that is, $F \subseteq \bigcap_{q \in A, g \in B} q(U-g) \in \mathcal{U}^{A, B}$.

We need one more lemma. Denote the collection of countable Borel good covers of a set $X$ by $\mathcal{B}_{\text {good }}$.

Lemma 13. If $|X|<\operatorname{cov}(\mathcal{M})$, then $X$ satisfies $\mathrm{S}_{1}\left(\mathcal{B}_{\text {good }}, \mathcal{B}_{\text {good }}\right)$.

Proof. Assume that $|X|<\operatorname{cov}(\mathcal{M})$, and let $\left\{\mathcal{U}_{n}\right\}_{n \in \mathbb{N}}$ be a sequence of countable Borel good covers of $X$. Enumerate each cover $\mathcal{U}_{n}$ by $\left\{U_{k}^{n}\right\}_{k \in \mathbb{N}}$. Let $\left\{Y_{n}\right\}_{n \in \mathbb{N}}$ be a partition of $\mathbb{N}$ into infinitely many infinite sets. For each $m$, let $y_{m} \in{ }^{\mathbb{N}}$ be an increasing enumeration of $Y_{m}$. Let $\left\{\mathcal{F}_{n}\right\}_{n \in \mathbb{N}}$ be an enumeration of all finite families of nonempty basic open sets.

For finite sets $F, B \subseteq X$ and $A \subseteq \mathbb{Q} \backslash\{0\}$, and each $m$ define a function $\Psi_{F, m}^{A, B} \in{ }^{\mathbb{N}} \mathbb{N}$ by

$\Psi_{F, m}^{A, B}(n)=\min \left\{k: F \subseteq V:=\bigcap_{q \in A, g \in B} q\left(U_{k}^{y_{m}(n)}-g\right)\right.$ and $\left.\left(\forall O \in \mathcal{F}_{m}\right) V \cap O \notin \mathcal{M}\right\}$.

Since there are less than $\operatorname{cov}(\mathcal{M})$ many functions $\Psi_{F, m}^{A, B}$, there exists by $[3]$ a function $f \in{ }^{\mathbb{N}} \mathbb{N}$ such that for each $m, F, A$, and $B, \Psi_{F, m}^{A, B}(n)=f(n)$ for infinitely many $n$. Consequently, $\mathcal{V}=\left\{U_{f(n)}^{y_{m}(n)}: m, n \in \mathbb{N}\right\}$ is a good cover of $X$.

We are now ready to carry out the construction. Let ${ }^{\mathbb{N}} \mathbb{R}=\left\{y_{\alpha}: \alpha<\mathfrak{c}\right\}$, $\left\{M_{\alpha}: \alpha<\mathfrak{c}\right\}$ be all $F_{\sigma}$ meager subsets of ${ }^{\mathbb{N}} \mathbb{R}$, and let $\left\{\left\{\mathcal{U}_{n}^{\alpha}\right\}_{n \in \mathbb{N}}: \alpha<\mathfrak{c}\right\}$ be all sequences of countable families of Borel sets. Let $\left\{O_{k}: k \in \mathbb{N}\right\}$ and $\left\{\mathcal{F}_{m}: m \in \mathbb{N}\right\}$ be all nonempty basic open sets and all finite families of nonempty basic open sets, respectively, in ${ }^{\mathbb{N}} \mathbb{R}$.

We construct $L_{1}$ and $L_{2}$ by induction on $\alpha<\mathfrak{c}$ as follows. At stage $\alpha \geq 0$ set $L_{\alpha}^{i}=\bigcup_{\beta<\alpha} L_{\beta}^{i}$ and consider the sequence $\left\{\mathcal{U}_{n}^{\alpha}\right\}_{n \in \mathbb{N}}$. Say that $\alpha$ is $i$-good if for 
each $n, \mathcal{U}_{n}^{\alpha}$ is a good cover of $L_{\alpha}^{i}$. In this case, by Lemma 13 there exist elements $U_{n}^{\alpha, i} \in \mathcal{U}_{n}^{\alpha}$ such that $\mathcal{U}^{\alpha, i}=\left\{U_{n}^{\alpha, i}\right\}_{n \in \mathbb{N}}$ is a good cover of $L_{\alpha}^{i}$. We make the inductive hypothesis that for each $i$-good $\beta<\alpha, \mathcal{U}^{\beta, i}$ is a good cover of $L_{\alpha}^{i}$. For finite sets $F, B \subseteq L_{\alpha}^{i}$ and $A \subseteq \mathbb{Q} \backslash\{0\}$, each $i$-good $\beta \leq \alpha$, and each $m$ define

$$
G_{A, B, F, m}^{\beta, i}=\bigcup\left(\left(\mathcal{U}^{\beta, i}\right)^{A, B}\right)_{F, \mathcal{F}_{m}} .
$$

As $\mathcal{U}^{\beta, i}$ is a good cover of $X_{\alpha}^{i},\left(\mathcal{U}^{\beta, i}\right)^{A, B}$ is $\omega$-fat cover of $L_{\alpha}^{i}$, and by Lemma 10, $G_{A, B, F, m}^{\beta, i}$ is comeager in ${ }^{\mathbb{N}} \mathbb{R}$. Set

$$
Y_{\alpha}=\bigcup_{\beta<\alpha} M_{\beta} \cup \bigcup\left\{\mathbb{N} \mathbb{R} \backslash G_{A, B, F, m}^{\beta, i}: \begin{array}{c}
i<2, i \text {-good } \beta \leq \alpha, m \in \mathbb{N}, \\
\text { finite sets } F, B \subseteq L_{\alpha}^{i}, A \subseteq \mathbb{Q} \backslash\{0\}
\end{array}\right\},
$$

and $Y_{\alpha}^{*}=\left\{x \in{ }^{\mathbb{N}} \mathbb{R}:\left(\exists y \in Y_{\alpha}\right) x={ }^{*} y\right\}$ (where $x={ }^{*} y$ means that $x(n)=y(n)$ for all but finitely many $n$ ). Then $Y_{\alpha}^{*}$ is a union of less than $\operatorname{cov}(\mathcal{M})$ many meager sets.

Lemma 14 (4]). If $X$ is a union of less than $\operatorname{cov}(\mathcal{M})$ many meager sets in ${ }^{\mathbb{N}} \mathbb{R}$, then for each $x \in{ }^{\mathbb{N}} \mathbb{R}$ there exist $y, z \in{ }^{\mathbb{N}} \mathbb{R} \backslash X$ such that $y+z=x$.

Use Lemma 14 to pick $x_{\alpha}^{0}, x_{\alpha}^{1} \in{ }^{\mathbb{N}} \mathbb{R} \backslash Y_{\alpha}^{*}$ such that $x_{\alpha}^{0}+x_{\alpha}^{1}=y_{\alpha}$. Let $k=$ $\alpha \bmod \omega$, and change a finite initial segment of $x_{\alpha}^{0}$ and $x_{\alpha}^{1}$ so that they both become members of $O_{k}$. Then $x_{\alpha}^{0}, x_{\alpha}^{1} \in O_{k} \backslash Y_{\alpha}$, and $x_{\alpha}^{0}+x_{\alpha}^{1}={ }^{*} y_{\alpha}$. Finally, define $X_{\alpha+1}^{i}=L_{\alpha}^{i}+\mathbb{Q} \cdot x_{\alpha}^{i}$. By Lemma 11, the inductive hypothesis is preserved. This completes the construction.

Take $L_{i}=\bigcup_{\alpha<\mathfrak{c}} L_{\alpha}^{i}, i=1,2$. By Lemma 13, each $L_{i}$ satisfies $\mathrm{S}_{1}\left(\mathcal{B}_{\text {good }}, \mathcal{B}_{\text {good }}\right)$, and by the construction, its intersection with each nonempty basic open set has size $\mathfrak{c}$. By Lemma 12, $\mathcal{B}_{\text {good }}=\mathcal{B}_{\Omega}$ for $L_{i}$. Finally, $L_{1}+L_{2}$ (a homomorphic image of $L_{1} \times L_{2}$ ) is a dominating subset of ${ }^{\mathbb{N}} \mathbb{R}$, thus $L_{1} \times L_{2}$ is not $o$-bounded.

Remark 15. None of the o-bounded groups $L_{1}$ and $L_{2}$ in Theorem 9 is strictly $o$-bounded. By an unpublished result of Michalewski, every metrizable strictly $o$ bounded group is a subgroup of a $\sigma$-compact group, and in [9, Theorem 5.3] it is proved that a product of such a group with an $o$-bounded group is $o$-bounded.

The following consequence of Theorem 9 seems nontrivial. Say that a subset $S$ of a topological group $G$ is $\aleph_{0}$-bounding if there exists a countable set $F \subseteq G$ such that $F \cdot S=G$. For example, $G$ is $\aleph_{0}$-bounded if each nonempty open set in $G$ is $\aleph_{0}$-bounding. The first property in the following corollary may be called Borel o-boundedness. This property is more interesting when the group in question is $\aleph_{0}$-bounded, in which case it is stronger than $o$-boundedness.

Corollary 16. Assume that $\operatorname{cov}(\mathcal{M})=\mathfrak{c}$. Then there exists an $\aleph_{0}$-bounded topological group $G$ of size continuum such that:

(1) For each sequence $\left\{B_{n}\right\}_{n \in \mathbb{N}}$ of $\aleph_{0}$-bounding Borel sets in $G$, there exists a sequence $\left\{F_{n}\right\}_{n \in \mathbb{N}}$ of finite subsets of $G$ such that $G=\bigcup_{n} F_{n} \cdot B_{n}$.

(2) Moreover, the sequence in (1) will have the property that for each finite $F \subseteq G$ there exists $n$ such that $F \subseteq F_{n} \cdot U_{n}$.

Remark 17. Banakh, Nickolas, and Sanchis have also, independently, proved the consistency of $o$-bounded groups not being closed under taking finite products (however, they do not consider stronger combinatorial properties as done in 13 and here). Their construction uses ultrafilters which are not nearly coherent; see [2]. 


\section{Groups satisfying $\mathrm{S}_{1}\left(\mathcal{B}_{\Omega}, \mathcal{B}_{\Gamma}\right)$ or $\mathrm{S}_{1}\left(\mathcal{B}_{\Omega}, \mathcal{B}_{\Gamma}\right)$}

One may wonder whether Theorem 9 can be strengthened further so that $L_{1}$ and $L_{2}$ will satisfy a stronger property. By inspection of the Scheepers Diagram 1. the only candidate for a stronger property (among the ones considered in this paper) is $S_{1}\left(\mathcal{B}_{\Omega}, \mathcal{B}_{\Gamma}\right)$. This is far from possible: A result of [1] (see [16, Theorem 32]) implies that whenever $G_{1}$ is a topological group satisfying $\mathrm{S}_{1}(\Omega, \Gamma)$, and $G_{2}$ is o-bounded, the group $G_{1} \times G_{2}$ is o-bounded. However strong, though, the notion of a topological group satisfying $\mathrm{S}_{1}\left(\mathcal{B}_{\Omega}, \mathcal{B}_{\Gamma}\right)$ is not trivial.

Theorem 18. For each cardinal $\kappa$ with $\mathrm{cf}(\kappa)>\aleph_{0}$, it is consistent that $\mathfrak{c}=\kappa$ and there exists a topological subgroup of $\mathbb{R}$ of size $\mathfrak{c}$, which satisfies $\mathrm{S}_{1}\left(\mathcal{B}_{\Omega}, \mathcal{B}_{\Gamma}\right)$.

Proof. This is really a theorem of Miller: Let $M$ be a countable standard model of ZFC satisfying $\mathfrak{c}=\kappa$. In [14] it is proved that there exists a ccc poset $\mathbb{P}$ in $M$ of size continuum (so that forcing with $\mathbb{P}$ does not change the size of the continuum) such that the old reals $M \cap \mathbb{R}$ satisfy $\mathrm{S}_{1}\left(\mathcal{B}_{\Omega}, \mathcal{B}_{\Gamma}\right)$ in $V^{\mathbb{P}}$.

But observe that, as the operations of addition and substraction in $\mathbb{R}$ are absolute, $G=M \cap \mathbb{R}$ is a group in $V^{\mathbb{P}}$.

Problem 19. Does the Continuum Hypothesis imply the existence of a separable metrizable group of size $\mathfrak{c}$ which satisfies $\mathrm{S}_{1}\left(\mathcal{B}_{\Omega}, \mathcal{B}_{\Gamma}\right)$ ?

We have some approximate results. For a sequence $\left\{X_{n}\right\}_{n \in \mathbb{N}}$ of subsets of $X$, define $\lim \inf X_{n}=\bigcup_{m} \bigcap_{n \geq m} X_{n}$. For a family $\mathcal{F}$ of subsets of $X, L(\mathcal{F})$ denotes its closure under the operation liminf. According to $[8, X$ is a $\delta$-set if for each $\omega$-cover $\mathcal{U}$ of $X, X \in L(\mathcal{U})$. It is easy to see that the $\delta$-property is preserved under taking countable increasing unions. Clearly $\mathrm{S}_{1}(\Omega, \Gamma)$ implies the $\delta$-property. The converse is still an open problem [19. If a $\delta$-set is a group, we will call it a $\delta$-group.

Let $\mathbb{Z}_{2}$ denote the usual group $\{0,1\}$ with modulo 2 addition.

Theorem 20. Assume that $\mathfrak{p}=\mathfrak{c}$. Then there exists a subgroup $G$ of $\mathbb{N}_{2}$ such that for each $k G^{k}$ is a countable increasing union of sets satisfying $\mathrm{S}_{1}(\Omega, \Gamma)$. In particular, all finite powers of $G$ are $\delta$-groups.

Proof. As $\mathfrak{p}=\mathfrak{c}$, there exists a subset $X$ of ${ }^{\mathbb{N}} \mathbb{Z}_{2}$ of size continuum which satisfies $\mathrm{S}_{1}(\Omega, \Gamma)[7$. We may assume that $0 \in X$. Consequently,

$$
G:=\langle X\rangle=\bigcup_{n \in \mathbb{N}}\left\{x_{1}+\cdots+x_{n}: x_{1}, \ldots, x_{n} \in X\right\}
$$

is a countable increasing union of continuous images of powers of $X$. But the property $S_{1}(\Omega, \Gamma)$ is closed under taking finite powers and continuous images [11. Observe that each finite power of $G$ is the countable increasing union of the same power of the original sets.

Assuming the Continuum Hypothesis, there exists a set of reals $X$ of size $\mathfrak{c}$ satisfying $\mathrm{S}_{1}\left(\mathcal{B}_{\Omega}, \mathcal{B}_{\Gamma}\right)$ (e.g., [14]). It is an open problem whether $\mathrm{S}_{1}\left(\mathcal{B}_{\Omega}, \mathcal{B}_{\Gamma}\right)$ is provably preserved under taking finite powers $[19$. If it is, then we have a positive answer to Problem 19 .

Theorem 21. Assume that $X \subseteq{ }^{\mathbb{N}} \mathbb{Z}_{2}$ satisfies $\mathrm{S}_{1}\left(\mathcal{B}_{\Omega}, \mathcal{B}_{\Gamma}\right)$ in all finite powers. Then $G=\langle X\rangle$ satisfies $\mathrm{S}_{1}\left(\mathcal{B}_{\Omega}, \mathcal{B}_{\Gamma}\right)$ in all finite powers.

Proof. By the above arguments, it suffices to prove the following. 
Lemma 22. $\mathrm{S}_{1}\left(\mathcal{B}_{\Omega}, \mathcal{B}_{\Gamma}\right)$ is preserved under taking countable increasing unions.

To prove the lemma, assume that $X=\bigcup_{n} X_{n}$ is an increasing union, and observe that $\mathrm{S}_{1}\left(\mathcal{B}_{\Omega}, \mathcal{B}_{\Gamma}\right)$ implies $\mathrm{S}_{1}\left(\mathcal{B}_{\Gamma}, \mathcal{B}_{\Gamma}\right)$, which in turn implies that all Borel images of each $X_{k}$ in ${ }^{\mathbb{N}} \mathbb{N}$ are bounded [17.

Assume that $\mathcal{U}_{n}=\left\{U_{m}^{n}: m \in \mathbb{N}\right\}, n \in \mathbb{N}$, are countable Borel $\omega$-covers of $X$. By $\mathrm{S}_{1}\left(\mathcal{B}_{\Omega}, \mathcal{B}_{\Gamma}\right)$, we may assume that each $\mathcal{U}_{n}$ is a $\gamma$-cover of $X_{n}$. For each $k$, define a function $\Psi_{k}$ from $X_{k}$ to ${ }^{\mathbb{N}} \mathbb{N}$ so that for each $x$ and $n$,

$$
\Psi_{k}(x)(n)=\min \left\{i:(\forall m \geq i) x \in U_{m}^{n}\right\} .
$$

$\Psi_{k}$ is a Borel map, thus $\Psi_{k}[X]$ is bounded. Consequently, $\bigcup_{k} \Psi_{k}[X]$ is bounded, say by the sequence $m_{n}$. Then $\left\{U_{m_{n}}^{n}\right\}_{n \in \mathbb{N}}$ is a $\gamma$-cover of $X$, as required.

We conclude the paper with a group satisfying $\mathrm{S}_{1}\left(\mathcal{B}_{\Gamma}, \mathcal{B}_{\Gamma}\right)$ in all finite powers. Let $\mathcal{N}$ denote the collection of null (Lebesgue measure zero) sets of reals. $\operatorname{cov}(\mathcal{N})$ is the minimal size of a cover of $\mathbb{R}$ by null sets, and $\operatorname{cof}(\mathcal{N})$ is the minimal size of a cofinal family in $\mathcal{N}$ with respect to inclusion. Let $\kappa$ be an uncountable cardinal. $S \subseteq \mathbb{R}$ is a $\kappa$-Sierpiński set if $|S| \geq \kappa$ and for each null set $N,|S \cap N|<\kappa$. b-Sierpiński sets satisfy $\mathrm{S}_{1}\left(\mathcal{B}_{\Gamma}, \mathcal{B}_{\Gamma}\right)$, but powers of $\kappa$-Sierpiński sets are never $\kappa$-Sierpiński sets.

Theorem 23. Assume that $\operatorname{cov}(\mathcal{N})=\operatorname{cof}(\mathcal{N})=\mathfrak{b}$. Then there exists a (non- $\sigma$ compact) group $G \subseteq \mathbb{R}$ of size $\mathfrak{b}$, such that all finite powers of $G$ satisfy $\mathrm{S}_{1}\left(\mathcal{B}_{\Gamma}, \mathcal{B}_{\Gamma}\right)$. Consequently, all finite powers of $G$ also satisfy $\mathrm{S}_{\text {fin }}\left(\mathcal{B}_{\Omega}, \mathcal{B}_{\Omega}\right)$.

Proof. The proof is similar to that of Theorem 7.2 of [6].

Lemma 24. Assume that $\operatorname{cov}(\mathcal{N})=\operatorname{cof}(\mathcal{N})$. Then there exists a $\operatorname{cov}(\mathcal{N})$-Sierpinski set $S$ such that for each $k$ and each null set $N$ in $\mathbb{R}^{k}, S^{k} \cap N$ is contained in a union of less than $\operatorname{cov}(\mathcal{N})$ many continuous images of $S^{k-1}$.

Proof. Let $\kappa=\operatorname{cov}(\mathcal{N})=\operatorname{cof}(\mathcal{N})$. Let $\left\{N_{\alpha}^{(k)}: \alpha<\kappa\right\}$ be a cofinal family of null sets in $\mathbb{R}^{k}, k \in \mathbb{N}$.

For $J \subseteq \mathbb{R}^{k}, x \in \mathbb{R}$, and $i<k$, define $J_{(x, i)}=\left\{v_{1} \frown v_{2}: \ell\left(v_{1}\right)=i, v_{1} \frown x \frown\right.$ $\left.v_{2} \in J\right\}$. By the Fubini Theorem, for each null set $N \subseteq \mathbb{R}^{k}$ and $i<k$,

$$
\tilde{N}=\left\{x:(\exists i<k) N_{(x, i)} \text { is not null in } \mathbb{R}^{k-1}\right\} \in \mathcal{N} .
$$

We make an inductive construction on $\alpha<\kappa$ of elements $x_{\alpha} \in \mathbb{R}$ with auxiliary collections $\mathcal{F}_{\alpha}$ of null sets, as follows. For $\alpha<\kappa$ let $\mathcal{P}_{\alpha}=\left\{N_{\alpha}^{(k)}: k \in \mathbb{N}\right\}$. At step $\alpha$ do the following:

(1) Choose $x_{\alpha} \notin \bigcup_{\beta<\alpha}\left(\bigcup_{N \in\left(\mathcal{P}_{\beta} \cup \mathcal{F}_{\beta}\right) \backslash P(\mathbb{R})} \tilde{N} \cup \bigcup_{N \in\left(\mathcal{P}_{\beta} \cup \mathcal{F}_{\beta}\right) \cap P(\mathbb{R})} N\right)$.

(2) Set $\mathcal{F}_{\alpha}=\left\{N_{\left(x_{\alpha}, i\right)}: \beta<\alpha, N \in\left(\mathcal{P}_{\beta} \cup \mathcal{F}_{\beta}\right) \backslash P(\mathbb{R}), i \in \mathbb{N}\right\}$.

This is possible because $x_{\alpha}$ is required to avoid membership in a union of less than $\operatorname{cov}(\mathcal{N})$ many null sets.

Take $S=\left\{x_{\alpha}: \alpha<\kappa\right\}$. Then $S$ is a $\kappa$-Sierpiński set. Fix $k$. For each null $N \subseteq \mathbb{R}^{k}$, there exists $\beta<\kappa$ with $N \subseteq N_{\beta}^{(k)}$. Whenever $\beta<\alpha_{0}<\cdots<$ $\alpha_{k-1}$, and $\pi$ is a permutation on $\{0, \ldots, k-1\},\left(N_{\beta}^{(k)}\right)_{\left(x_{\alpha_{0}}, \pi^{-1}(0)\right)} \in \mathcal{F}_{\alpha_{0}}$, thus $\left(N_{\beta}^{(k)}\right)_{\left(x_{\alpha_{0}}, \pi^{-1}(0)\right),\left(x_{\alpha_{1}}, \pi^{-1}(1)\right)} \in \mathcal{F}_{\alpha_{1}}, \ldots,\left(N_{\beta}^{(k)}\right)_{\left(x_{\alpha_{0}}, \pi^{-1}(0)\right), \ldots,\left(x_{\left.\alpha_{k-2}, \pi^{-1}(k-2)\right)}\right.} \in$ $\mathcal{F}_{\alpha_{k-2}}$, thus $x_{\alpha_{k-1}} \notin\left(N_{\beta}^{(k)}\right)_{\left(x_{\alpha_{0}}, \pi^{-1}(0)\right), \ldots,\left(x_{\alpha_{k-2}}, \pi^{-1}(k-2)\right)}$, that is, $\left(x_{\alpha_{\pi(0)}}, \ldots\right.$, $\left.x_{\alpha_{\pi(k-1)}}\right) \notin N_{\beta}^{(k)}$. 
Consequently, $S^{k} \cap N$ is contained in the union of all sets of the form $S^{i} \times\left\{x_{\xi}\right.$ : $\xi \leq \beta\} \times S^{k-i-1}(i<k)$-a union of $|\beta|<\kappa$ copies of $S^{k-1}$ —and $\left\{v \in S^{k}: v_{i}=v_{j}\right\}$ $(i<j<k)$, which are continuous images of $S^{k-1}$.

Now assume that $\operatorname{cov}(\mathcal{N})=\operatorname{cof}(\mathcal{N})=\mathfrak{b}$, and let $S$ be a $\mathfrak{b}$-Sierpiński set as in Lemma 24. We will show by induction that for each $k, S^{k}$ satisfies $\mathrm{S}_{1}\left(\mathcal{B}_{\Gamma}, \mathcal{B}_{\Gamma}\right)$. By [17] it is enough to show that for each null $N \subseteq \mathbb{R}^{k}, S^{k} \cap N$ satisfies $\mathrm{S}_{1}\left(\mathcal{B}_{\Gamma}, \mathcal{B}_{\Gamma}\right)$. By Lemma 24 and the induction hypothesis, $S^{k} \cap N$ is contained in a union of less than $\mathfrak{b}$ many sets satisfying $\mathrm{S}_{1}\left(\mathcal{B}_{\Gamma}, \mathcal{B}_{\Gamma}\right)$. In [4, full version] it is shown that $\mathrm{S}_{1}\left(\mathcal{B}_{\Gamma}, \mathcal{B}_{\Gamma}\right)$ is preserved under taking unions of size less than $\mathfrak{b}$, and in [5] it is shown that $\mathrm{S}_{1}\left(\mathcal{B}_{\Gamma}, \mathcal{B}_{\Gamma}\right)$ is preserved under taking subsets. This proves the assertion.

So all finite powers of $S$ satisfy $\mathrm{S}_{1}\left(\mathcal{B}_{\Gamma}, \mathcal{B}_{\Gamma}\right)$, and $G=\langle S\rangle$ works, since as we mentioned before, $S_{1}\left(\mathcal{B}_{\Gamma}, \mathcal{B}_{\Gamma}\right)$ is preserved under taking countable unions. Finally, by [17. $\mathrm{S}_{1}\left(\mathcal{B}_{\Gamma}, \mathcal{B}\right)$ in all finite powers implies $\mathrm{S}_{\text {fin }}\left(\mathcal{B}_{\Omega}, \mathcal{B}_{\Omega}\right)$.

It seems that the following was not known before.

Corollary 25. Assume that $\operatorname{cov}(\mathcal{N})=\operatorname{cof}(\mathcal{N})=\mathfrak{b}$. Then there exists a set of reals satisfying $\mathrm{S}_{1}\left(\mathcal{B}_{\Gamma}, \mathcal{B}_{\Gamma}\right)$ and $\mathrm{S}_{\text {fin }}\left(\mathcal{B}_{\Omega}, \mathcal{B}_{\Omega}\right)$, but not $\mathrm{S}_{1}(\mathcal{O}, \mathcal{O})$.

\section{REFERENCES}

[1] L. Babinkostova, Lj. D. R. Kočinac, and M. Scheepers, Combinatorics of open covers (XI): Topological groups, in progress.L. Babinkostova, Lj. D. R. Kočinac, and M. Scheepers, Combinatorics of open covers (XI): Topological groups, in progress.

[2] T. Banakh, P. Nickolas, and M. Sanchis, Filter games and pathological subgroups of a countable product of lines, Journal of the Australian Mathematical Society, to appear.

[3] T. Bartoszyński, Combinatorial aspects of measure and category, Fundamenta Mathematicae 127 (1987), 209-213. MR0917147 (88m:04001)

[4] T. Bartoszyński, S. Shelah, and B. Tsaban, Additivity properties of topological diagonalizations, Journal of Symbolic Logic 68 (2003), 1254-1260. (Full version: http://arxiv.org/abs/math.Lo/0112262) MR2017353 (2004k:03094)

[5] T. Bartoszyński and B. Tsaban, Hereditary topological diagonalizations and the MengerHurewicz Conjectures, Proceedings of the American Mathematical Society, to appear.

[6] L. Bukovsky, I. Reclaw, M. Repicky, Spaces not distinguishing convergences of real-valued functions, Topology and its Applications 112 (2001), 13-40. MR1815270 (2002e:54010)

[7] F. Galvin and A. W. Miller, $\gamma$-sets and other singular sets of real numbers, Topology and its Applications 17 (1984), 145-155. MR0738943(85f:54011)

[8] J. Gerlits and Zs. Nagy, Some properties of $C(X)$, I, Topology and its Applications 14 (1982), 151-161. MR0667661 (84f:54021)

[9] C. Hernandez, Topological groups close to being $\sigma$-compact, Topology and its Applications 102 (2000), 101-111. MR 1739266 (2000k:54032)

[10] C. Hernandez, D. Robbie and M. Tkachenko, Some properties of o-bounded and strictly o-bounded groups, Applied General Topology 1 (2000), 29-43. MR 1796930 (2001g:22002)

[11] W. Just, A. W. Miller, M. Scheepers, and P. J. Szeptycki, The combinatorics of open covers II, Topology and its Applications 73 (1996), 241-266. MR1419798 (98g:03115a)

[12] A. Krawczyk and H. Michalewski, An example of a topological group, Topology and its Applications 127 (2003), 325-330. MR1941171 (2003j:54034)

[13] A. Krawczyk and H. Michalewski, Linear metric spaces close to being $\sigma$-compact, Technical Report 46 (2001) of the Institute of Mathematics, Warsaw University. http://www.minuw . edu.pl/english/research/reports/tr-imat/46/products.ps

[14] A. W. Miller, A Nonhereditary Borel-cover $\gamma$-set, Real Analysis Exchange 29 (2003/4), 601606. MR2083799

[15] M. Scheepers, Combinatorics of open covers I: Ramsey theory, Topology and its Applications 69 (1996), 31-62. MR1378387 (97h:90123)

[16] M. Scheepers, Selection principles and covering properties in topology, Note di Matematica 22 (2003), 3-41. 
[17] M. Scheepers and B. Tsaban, The combinatorics of Borel covers, Topology and its Applications 121 (2002), 357-382. MR1908999 (2003e:03091)

[18] M. Tkačenko, Introduction to topological groups, Topology and its Applications 86 (1998), 179-231. MR1623960 (99b:54064)

[19] B. Tsaban, Selection principles in Mathematics: A milestone of open problems, Note di Matematica 22 (2003), 179-208.

Department of Applied Mathematics and Computer Science, The Weizmann Institute of Science, Rehovot 76100, Israel

E-mail address: boaz.tsaban@weizmann.ac.il

$U R L:$ http://www.cs.biu.ac.il/ tsaban 